\title{
Substrate and Transition State Binding in Alkaline Phosphatase Analyzed by Computation of Oxygen Isotope Effects
}

\author{
Daniel Roston* and Qiang Cui* \\ Department of Chemistry and Theoretical Chemistry Institute, University of Wisconsin, \\ Madison, WI 53706
}

Email: droston@chem.wisc.edu,cui@chem.wisc.edu

\section{Supporting Information}

\section{Text S1}

Rate equations for the AP-catalyzed reaction are derived based on the kinetic mechanism shown in Scheme S1.1,2

Scheme S1: The kinetic mechanism of AP.

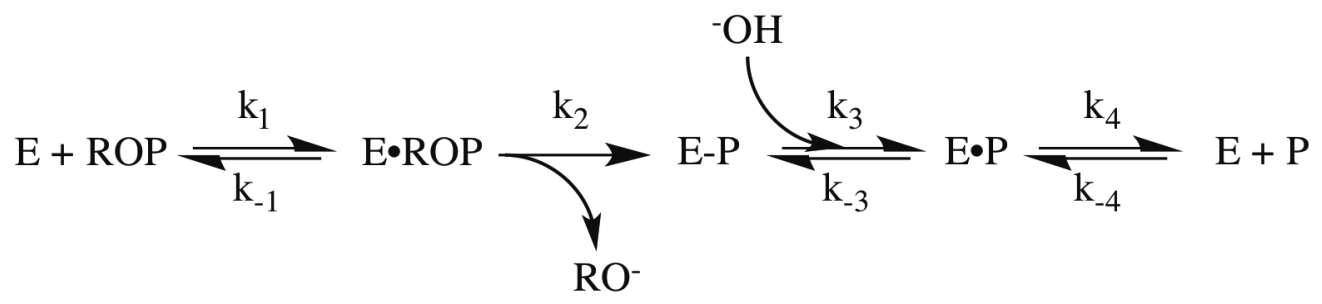

Based on this mechanism one can derive an overall rate $(v)$ as

$v=\frac{k_{c a t}[R O P][E]_{T}}{[R O P]+K_{I}[P]+K_{M}[R O P]}$

where the parameters $\mathrm{k}_{\mathrm{cat}}, \mathrm{K}_{\mathrm{M}}$, and $\mathrm{K}_{\mathrm{I}}$ are defined as follows:

$k_{\text {cat }}=\frac{k_{2} k_{3} k_{4}}{k_{2} k_{-3}+k_{3} k_{4}+k_{2}\left(k_{3}+k_{4}\right)}$

$K_{M}=\frac{k_{3} k_{4}\left(k_{2}+k_{-1}\right)}{k_{1}\left(k_{2} k_{-3}+k_{3} k_{4}+k_{2}\left(k_{3}+k_{4}\right)\right)}$

$K_{I}=\frac{k_{-4}\left(k_{2}+k_{-1}\right)\left(k_{3}+k_{-3}\right)}{k_{1}\left(k_{2}\left(k_{3}+k_{-3}\right)+k_{4}\left(k_{2}+k_{3}\right)\right)}$ 
Since KIEs are measured using competitive methods, ${ }^{3}$ the measured values

correspond to KIEs on $\mathrm{V} / \mathrm{K}$ (i.e., $\mathrm{k}_{\text {cat }} / \mathrm{K}_{\mathrm{M}}$ ):4,5

$k_{\text {cat }} /_{K_{M}}=\frac{k_{1} k_{2}}{k_{2}+k_{-1}}$

Under conditions where the chemical step is slow $\left(\mathrm{k}_{2}<<\mathrm{k}_{-}\right)$, as is the case for alkyl

substrates in $\mathrm{WT}^{1}$ and all substrates in the R166S mutant, 2,6

$k_{\text {cat }} /_{K_{M}} \approx \frac{k_{1} k_{2}}{k_{-1}}=K_{\text {Bind }} \times k_{\text {chem }}$

S6

and the KIE on $\mathrm{V} / \mathrm{K}$ can be approximated as

${ }^{18}(V / K) \approx{ }^{18} K_{\text {Bind }} \times{ }^{18} k_{\text {chem }}$

S7

\section{Additional Results}

Table S1: KIEs on $k_{c h e m}$ calculated at the B3LYP level with $3 \mathrm{~N}-6$ and $3 \mathrm{~N}$ degrees of freedom. ${ }^{a}$

\begin{tabular}{lcc}
\hline Substrate & $3 \mathrm{~N}-6$ & $3 \mathrm{~N}$ \\
\hline m-nitrophenyl phosphate (mNPP) & 1.009907 & 1.009908 \\
phenyl phosphate (PhOP) & 1.008891 & 1.008894 \\
p-aminophenyl phosphate (pAPP) & 1.007038 & 1.007040 \\
propargyl phosphate (PrAP) & 1.009595 & 1.009596 \\
m-nitrobenzyl phosphate (mNBP) & 1.006984 & 1.006984 \\
ethyl phosphate (EtOP) & 1.009803 & 1.009815 \\
\hline aKIE calculations normally omit rotations and translations, leaving 3N-6 degrees of freedom. Williams ${ }^{7}$ recently advised \\
including all degrees of freedom in "cut-off" models where the system being analyzed is not strictly speaking at a stationary \\
point of its potential energy surface.
\end{tabular}

Table S2: EIEs for phosphate mono- and diesters using different solvent models.

\begin{tabular}{|c|c|c|c|c|c|c|c|c|}
\hline & & & $\mathrm{PCM}$ & & & SMD & & Explicit Solvent \\
\hline Substrate & $\mathrm{LG} \mathrm{pK}_{\mathrm{a}}$ & B3LYP & M06 & PBE & B3LYP & M06 & PBE & DFTB3/MM \\
\hline \multicolumn{9}{|l|}{ Monoesters } \\
\hline m-nitrophenyl phosphate (mNPP) & 8.4 & 1.016 & 1.018 & 1.012 & 1.020 & 1.022 & 1.014 & 1.014 \\
\hline phenyl phosphate (PhOP) & 10.0 & 1.019 & 1.019 & 1.014 & 1.028 & 1.028 & 1.023 & 1.014 \\
\hline p-aminophenyl phosphate (pAPP) & 10.3 & 1.019 & 1.022 & 1.014 & 1.034 & 1.032 & 1.029 & 1.018 \\
\hline propargyl phosphate (PrAP) & 13.6 & 1.034 & 1.035 & 1.027 & 1.024 & 1.045 & 1.016 & 1.021 \\
\hline m-nitrobenzyl phosphate (mNBP) & 14.9 & 1.034 & 1.037 & 1.028 & 1.021 & 1.038 & 1.015 & 1.023 \\
\hline ethyl phosphate (EtOP) & 16.0 & 1.035 & 1.038 & 1.030 & 1.022 & 1.036 & 1.014 & 1.023 \\
\hline \multicolumn{9}{|l|}{ Diesters } \\
\hline methyl-p-nitrophenyl phosphate (mpNPP) & 7.2 & 1.014 & 1.019 & 1.014 & 1.022 & 1.024 & 1.018 & 1.008 \\
\hline methyl-m-nitrophenyl phosphate (mmNPP) & 8.4 & 1.023 & 1.025 & 1.018 & 1.030 & 1.031 & 1.025 & 1.012 \\
\hline methyl-phenyl phosphate (mPhOP) & 10.0 & 1.025 & 1.028 & 1.020 & 1.040 & 1.039 & 1.035 & 1.017 \\
\hline
\end{tabular}


Table S3: Change in Mulliken charge on $\mathrm{O}_{\lg }$ upon substrate binding in WT AP.

\begin{tabular}{ll}
\hline Substrate & $\Delta \mathrm{Q}^{\mathrm{a}}$ \\
\hline mNPP & -0.02 \\
PhOP & -0.05 \\
pAPP & -0.04 \\
PrAP & -0.02 \\
EtOP & -0.04 \\
\hline${ }^{a}$ Difference between bound and unbound Mulliken charge at the DFTB3 level, calculated as the average of 1000 frames from \\
trajectories in each state.
\end{tabular}

\section{References}

(1) O'Brien, P. J.; Herschlag, D. Biochemistry 2002, 41, 3207.

(2) O'Brien, P. J.; Lassila, J. K.; Fenn, T. D.; Zalatan, J. G.; Herschlag, D. Biochemistry 2008, 47, 7663.

(3) Zalatan, J. G.; Catrina, I.; Mitchell, R.; Grzyska, P. K.; O'Brien, P. J.;

Herschlag, D.; Hengge, A. C. J. Am. Chem. Soc. 2007, 129, 9789.

(4) Hengge, A. C. Accounts Chem. Res. 2002, 35, 105.

(5) Cook, P. F.; Cleland, W. W. Enzyme kinetics and mechanism; Taylor \& Francis Group: New York, 2007.

(6) O'Brien, P. J.; Herschlag, D. J. Am. Chem. Soc. 1999, 121, 11022.

(7) Williams, I. H.J. Chem. Theory Comput. 2012, 8, 542. 